\title{
Introdução e condução dos métodos mistos de pesquisa em educação física
}

\section{Introduction and conduct of mixed research methods in physical education}

\section{Introducción y conducta de métodos de investigación mixta en educación física}

\author{
iD (9) Mariane Ferreira \\ Universidade Federal de São Paulo, Santos, São Paulo, Brasil \\ E-mail: ma_ferreira2@hotmail.com \\ iD (9) João Francisco Barbieri \\ Universidade Estadual de Campinas, Campinas, São Paulo, Brasil \\ E-mail: francisco600_25@hotmail.com \\ iD 9 \\ José Julio Gavião de Almeida \\ Universidade Estadual de Campinas, Campinas, São Paulo, Brasil \\ E-mail: gaviao@fef.unicamp.br
}

iD Ciro Winckler

Universidade Federal de São Paulo, Santos, São Paulo, Brasil

E-mail ciro.winckler@unifesp.br

\begin{abstract}
Resumo: Os métodos mistos de pesquisa são definidos como um processo de recolhimento, análise e "mistura" de dados quantitativos e qualitativos durante determinado estágio da pesquisa em um único estudo. $\mathrm{Na}$ área da Educação Física, esse método de pesquisa é uma combinação de abordagens relativamente nova. Assim, o presente ensaio teve como objetivo construir orientações fundamentais para o desenvolvimento de estudos de métodos mistos na área da Educação Física. Conclui-se, pois, que a busca pelo entendimento de como se dá o desenvolvimento de um estudo de métodos mistos pode auxiliar o pesquisador diante das
\end{abstract}


construções de pesquisas, além de trazer contribuições para os avanços científicos.

Palavras-chave: Pesquisa. Pesquisa mista. Desenho de pesquisa. Educação Física.

\begin{abstract}
Mixed research methods are defined as a process of collecting, analyzing and "mixing" quantitative and qualitative data during a given stage of research and in a single study. In the area of Physical Education, this research method is a relatively new combination of approaches. Thus, this essay aimed to build fundamental orientations for the development of mixed methods studies in the Physical Education area. It is concluded, therefore, that the search for the understanding of how the development of a mixed methods study takes place can help the researcher in the face of the research constructions, besides bringing contributions to the scientific advances.
\end{abstract}

Keywords: Research. Mixed research. Research design. Physical Education.

Resumen: Los métodos de investigación mixtos se definen como un proceso de recopilación, análisis y "mezcla" de datos cuantitativos y cualitativos durante una etapa dada de investigación y en un solo estudio. En el área de Educación Física, este método de investigación es una combinación relativamente nueva de enfoques. Por lo tanto, este ensayo tuvo como objetivo construir orientaciones fundamentales para el desarrollo de estudios de métodos mixtos en el área de Educación Física. Se concluye, por lo tanto, que la búsqueda de la comprensión de cómo tiene lugar el desarrollo de un estudio de métodos mixtos puede ayudar al investigador frente a las construcciones de investigación, además de aportar contribuciones a los avances científicos.

Palabras clave: Investigación. Investigación mixta. Diseño de investigación. Educación Física.

Submetido em: 11-08-2019

Aceito em: 06-04-2020 


\section{1 introdução}

Os métodos mistos de pesquisa são definidos como um processo de recolhimento, análise e "mistura" de dados quantitativos e qualitativos durante determinado estágio da pesquisa em um único estudo. O método misto tem por objetivo compreender melhor o problema de pesquisa (TASHAKKORI e TEDDLIE 2003; CRESWELL 2005). Nessa perspectiva, sabe-se que os métodos mistos de pesquisa vêm sendo discutidos desde a década de 1970 nas mais diversas áreas (CRESWELL, 2003).

As primeiras publicações que descreveram e definiram a metodologia mista são datadas do final da década de 1980 (CRESWELL e CLARK, 2015). Depois desse período, estudos foram modelados a partir dessa nova perspectiva de pesquisa. De acordo com Leech e Onweugbuzie (2009), a maioria dos estudos de metodologia mista tem sido utilizada para responder perguntas nas quais um único paradigma não alcançaria respostas. Como exemplo de pesquisa mista, identifica-se um estudo realizado por Caregnato, Ordonhes e Cavichiolli (2018), no qual os autores analisaram a estrutura física do atletismo no Brasil e identificaram, ainda, as principais entidades que promoveram essa modalidade no país. Assim, foi realizado um estudo documental, conduzido por uma intervenção qualitativa, a partir da Análise de Conteúdo, sobre os dados da busca documental, além de uma análise quantitativa das variáveis identificadas através dessa busca.

Creswell, Fetters e Ivankova (2004) afirmam que a ideia subjacente da metodologia mista indica que nenhum dos métodos, quantitativos e qualitativos, são suficientes por si só, pois podem não alcançar as tendências e os detalhes do objeto de estudo. Com a utilização dos métodos mistos, é possível produzir análises mais completas do problema pesquisado. Dessa maneira, espera-se que a combinação dos métodos seja feita para que eles se complementem mutuamente (CRESWELL, FETTERS e IVANKOVA, 2004). 
Atualmente, a ideia que define os métodos mistos tem incorporado diferentes pontos de vista (CRESWELL e CLARK, 2015). Assim, o presente estudo buscou focar nas características essenciais para o desenvolvimento de pesquisas baseadas no método misto na área da Educação Física. Thomas, Nelson e Silverman (2012) afirmam que, nessa área, o método misto de pesquisa trata-se de uma combinação de abordagens relativamente recente. De acordo com os autores, durante muito tempo, os acadêmicos de diversas áreas de pesquisa acreditavam ser impraticável esse tipo de combinação em um mesmo estudo.

Nesse sentido, o presente ensaio teve como objetivo construir orientações fundamentais para o desenvolvimento de estudos de métodos mistos na área da Educação Física. Para tanto, serão apresentados: a) os conceitos dos métodos de pesquisa mista e b) os caminhos necessários para selecionar o design de métodos mistos apropriados para a realização de um estudo de métodos mistos na área da Educação Física.

\section{Delineamento de pesquisa de método misto}

Segundo Thomas, Nelson e Silverman (2012), a pesquisa de método misto é pragmática, na qual o método selecionado é influenciado pelas perguntas. O pragmatismo é definido como fenômeno consequente, ou seja, trata-se das possibilidades da ação que só são uteis quando produzem resultados práticos (DEWEY, 1931). Portanto, o pragmatismo está, de maneira geral, associado à pesquisa de métodos mistos, pois foca nas consequências da pesquisa, de modo a responder, amplamente, a questão elaborada, além de destacar os métodos dados para elucidar, de maneira sistêmica, o problema estudado (CRESWELL e CLARCK, 2015). Alguns autores têm recorrido cada vez mais a esse modelo pragmático, por exemplo, Johnson e Onwuwgbuzie (2004); Rallis e Rossman (2003); Morgan (2007). Creswell e Clarck (2015, p. 53) afirmam que esses autores "têm se movido para identificar a 'melhor' 
visão de mundo que proporcione um fundamento para a pesquisa de métodos mistos".

Small (2011) afirma que, para alguns autores, o pragmatismo tem sido considerado um pouco além de um sinônimo para orientação prática, ou seja, compreende um sentido voltado ao abandono das perguntas abstratas de maneira que se favoreça uma abordagem mais concreta. Dessa forma, o que se entende é que há uma crença básica no pragmatismo, que oferece o suporte teórico apropriado ao priorizar as ações de descoberta da justificativa para o alcance do conhecimento (SMALL, 2011). O pesquisador pragmático preocupa-se, em primeiro lugar, com o quebra-cabeça empírico, resolvendo-o através do que for parecendo mais útil durante o processo (SMALL, 2011).

Essa orientação oferece, naturalmente, uma gama de espaço para as perspectivas metodológicas (SMALL, 2011). Por conseguinte, o pragmatismo é encarado como uma perspectiva transformativa, pois possibilita aos pesquisadores a adoção de uma postura pluralista, de maneira que as questões da pesquisa sejam respondidas por meio do alcance de todos os tipos de dados (CRESWELL e CLARCK, 2015). Tashakkori e Teddie (2003) argumentam que, dentre outros pontos, a questão da pesquisa é primordialmente importante. Essa importância é realçada, pois preocupa-se com o fundamento que as perguntas trarão ao estudo.

Portanto, pode-se afirmar que o método selecionado é influenciado pelas perguntas do estudo (THOMAS, NELSON e SILVERMAN, 2012). À vista disso, "um estudo pode ser essencialmente quantitativo e com uma parte que é qualitativa. Contudo, pode ser essencialmente qualitativo com um componente quantitativo" (THOMAS, NELSON e SILVERMAN, 2012, p. 392). Ridenour e Newman (2008) descrevem que, entre esses dois métodos, há um contínuo pelo qual os métodos mistos são representados de maneira holística para abordar a pesquisa. Para os autores, assumir o contínuo resulta em aceitar, como lugar central da teoria, que tem de haver uma coexistência apropriada de todos os outros componentes com relação ao contínuo (RIDENOUR e NEWMAN, 2008). 
Assim, ao utilizar o modelo e planejamento da pesquisa, de acordo com sua estrutura, possibilita, de maneira consistente, a realização de uma pesquisa sob ótima validade (RIDENOUR e NEWMAN, 2008). Os pesquisadores que assumem esse modelo focam em uma filosofia de pesquisa unificada e em um método consistente - não na ideologia (RIDENOUR e NEWMAN, 2008). Para conceituar a lógica diante da aplicação do contínuo, Ridenour e Newman (2008) sugerem três fases:

Primeira fase: o contínuo apresenta consistência entre a questão e o método, assim, a proposta e a pergunta da pesquisa serão as bases pelas quais os pesquisadores irão tomar a decisão pelo design experimental;

Segunda fase: é avaliado até que ponto a consistência existe. Dessa forma, é identificada essa consistência através da proposta do estudo, da questão da pesquisa e, por fim, dos métodos de pesquisa, pois esses pontos irão estabelecer a validade do estudo;

Terceira fase: análise de validade do design no estudo. Sendo assim, essa validade só irá ocorrer quando a estrutura organizada para a coleta de dados permitir medir a qualidade científica do desenho experimental.

Assim, essas fases conduzem o alcance do contínuo de pesquisa de métodos mistos apresentados por Thomas, Nelson e Silverman (2012), na figura 1. 
Figura 1 - Contínuo de pesquisa de métodos mistos

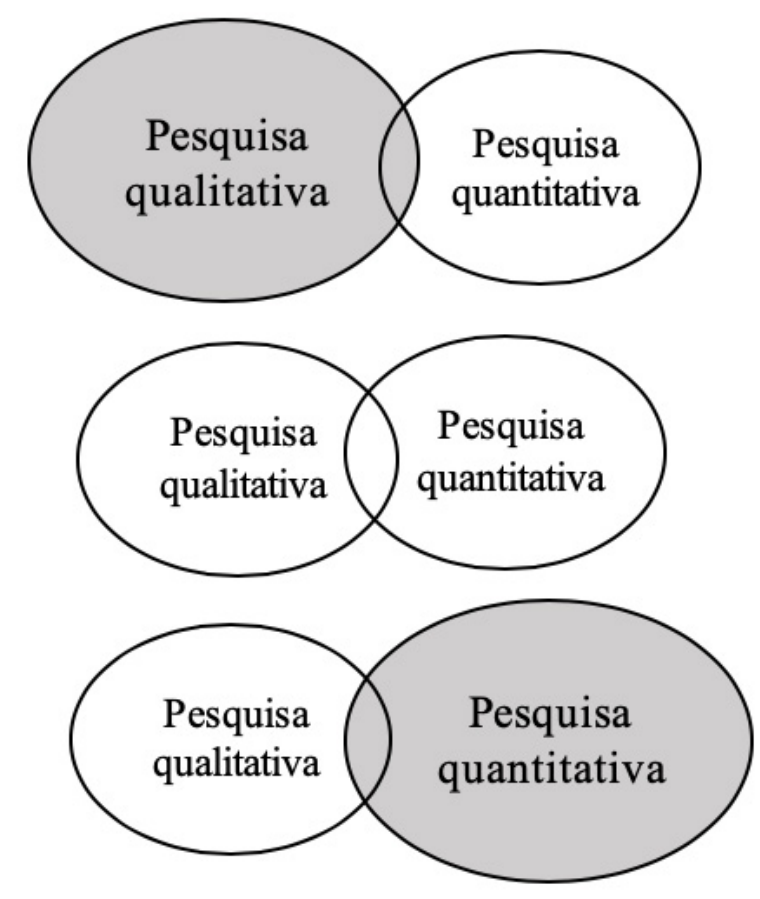

Fonte: Adaptado Thomas, Nelson e Silverman (2012).

Olhando para o contínuo de pesquisa de métodos mistos apresentado na figura 1, o que simboliza, em cada tipo, são os métodos específicos usados em cada parte da pesquisa, sendo que haverá um método dominante dependendo do modelo adotado para responder à pergunta do estudo (THOMAS, NELSON e SILVERMAN, 2012). Nesse sentido, o estudo de método misto pode coletar dados qualitativos com base nos dados quantitativos (THOMAS, NELSON e SILVERMAN, 2012). Em uma intervenção experimental, essa combinação pode ser realizada através de uma avaliação de percepção dos participantes, através de um estudo qualitativo (THOMAS, NELSON e SILVERMAN, 2012).

Um exemplo desse tipo de projeto é o estudo experimental realizado por Figueiras et al. (2012), no qual os autores buscaram compreender a aplicação da imagética mental diante do trabaIho do 'Psicólogo Esportivo' na demanda do desempenho de determinado atleta durante um campeonato. Para isso, os autores 
apresentaram o relato do técnico da equipe, com uma intervenção qualitativa, a fim de identificar, através da percepção do técnico, a postura e o comprometimento do jogador antes e após a intervenção, e teve ainda por base os dados quantitativos através da quantidade de gols marcados durante o campeonato e as cobranças de penalidades durante os treinamentos.

Outro exemplo trata-se de um estudo realizado por Jaarsma et al. (2014), no qual os autores buscaram identificar as barreiras e as facilidades para a participação esportiva de crianças com deficiência física. Assim, os dados quantitativos foram coletados através de um questionário com perguntas sobre a participação esportiva, deficiência física e as barreiras e facilidades da prática esportiva. Esse questionário foi aplicado aos pais e às crianças de uma escola de educação especial para crianças com deficiência física na Holanda. Os dados qualitativos foram coletados através de uma entrevista semiestruturada com os professores e profissionais da saúde dessa escola, abordando a participação nas práticas esportivas dos alunos com deficiência. Com isso, os autores do estudo fizeram a combinação dos dados dos questionários (quantitativo) com os dados extraídos da entrevista (qualitativo).

Thomas, Nelson e Silverman (2012, p. 392) descrevem que são infinitas as combinações dos delineamentos de métodos mistos, sendo que "qualquer combinação de questões que sugira métodos mistos pode ser acomodada, desde que o pesquisador ou 0 grupo de pesquisadores tenha a habilidade para conduzir todas as partes do estudo". Creswell e Clarck (2015) afirmam que é preciso perceber que os métodos mistos de pesquisa podem ser fixos e/ou emergentes, ou seja, é preciso ter conhecimento do tipo de abordagem a ser utilizada, pois o objetivo é considerar a melhor alternativa.

Assim, os projetos com métodos mistos fixos são abordagens planejadas no início da pesquisa, com a utilização dos métodos qualitativos e quantitativos (CRESWELL e CLARCK, 2015). Os projetos de métodos mistos emergentes, por sua vez, são aqueles em 
que os métodos mistos surgem durante o processo de condução da pesquisa (CRESWELL e CLARCK, 2015).

\subsection{Design dos métodos mistos de pesquisa}

O projeto de pesquisa mista envolve várias tipologias, entretanto existem seis tipos principais, sendo: três tipos sequenciais (explicativos, exploratórios e transformadores) e três tipos concorrentes (triangulação, aninhada e transformativa) (HANSON et al., 2005; CRESWELL et al., 2007). Cada um desses tipos varia de acordo com o uso de determinada teoria, da abordagem à implementação (sequência dos procedimentos de coleta de dados simultâneos), a prioridade que será dada aos dados quantitativos e aos dados qualitativos (iguais ou desiguais) e, ainda, o estágio em que os dados serão analisadose integrados (separados, transformados ou conectados) (HANSON et al., 2005).

Métodos mistos sequenciais: A abordagem escolhida difere na sequência de coleta de dados, que pode ser definida através de um design sequencial, e as condições metodológicas conduzem um dado a ser precedido pelo outro (TASHAKKORI e TEDDLIE, 1998; SMALL, 2011). Alguns estudos de métodos mistos têm explorado várias das vantagens dos estudos sequenciais, como, por exemplo, testar as hipóteses emergentes, ou, ainda, compreender a capacidade dos mecanismos por trás das associações recém-descobertas (SMALL, 2011). De acordo com Tashakkori e Teddlie (2003), existem três tipos de design sequenciais, sendo: sequencial explicativo, sequencial exploratório e sequencial transformativo.

Os projetos explicativos sequenciais elucidam os relacionamentos e/ou estudam os achados (HANSON et al., 2005). Assim, são coletados e analisados os dados quantitativos seguidos dos dados qualitativos, sendo que é desigual a prioridade, ou seja, são utilizados os dados qualitativos para aumentar os dados quantitativos, porém essa análise de dados é conectada, pois objetiva in- 
tegrar os dados selecionados na fase de interpretação e discussão (CRESWELL et al., 2003; HANSON et al., 2005). O esquema a seguir representa esse tipo de design sequencial, sendo que o símbolo ( ) indica a direção do processo. As letras em maiúsculo representam a dominância; e as letras em minúsculo representam o projeto suplementar (MORSE, 2003).

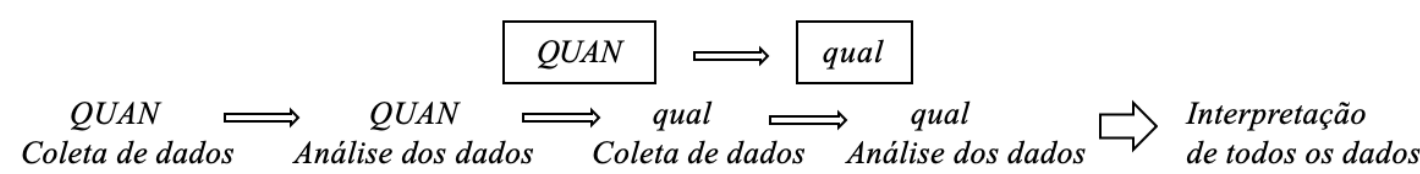

Fonte: adaptado de Creswell et al. (2003).

Os projetos exploratórios sequenciais possuem muitas características semelhantes aos projetos sequenciais explicativos (CRESWELL et al., 2003). Assim, primeiro são coletados e analisados os dados qualitativos; e, depois, os dados quantitativos, sendo que, de maneira geral, a prioridade é desigual (CRESWELL et al., 2003; HANSON et al., 2005). Dessa forma, os dados quantitativos são usados, principalmente, para o aumento dos dados qualitativos. A integração desses dados, por sua vez, é realizada na fase de interpretação e discussão (CRESWELL et al., 2003; HANSON et al., 2005). Esse design é útil quando são desconhecidas as variáveis do estudo, testando, pois, uma teoria emergente (HANSON et al., 2005).

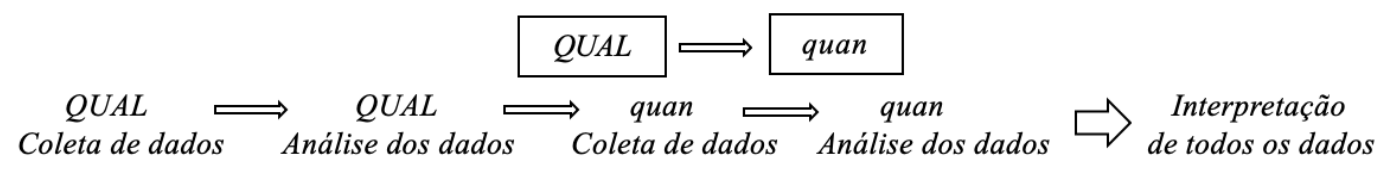

Fonte: adaptado de Creswell et al. (2003).

Em oposição, os projetos sequenciais transformadores geralmente refletem no propósito, nas questões de pesquisa e nas implicações de ações e mudanças (HANSON et al., 2005). Ainda, nesses projetos, os dados quantitativos podem ser coletados e 
analisados, seguidos pelos dados qualitativos ou o inverso. Dessa forma, qualquer tipo de dados pode ser coletado primeiro, dependendo da preferência das necessidades do pesquisador (HANSON et al., 2005).

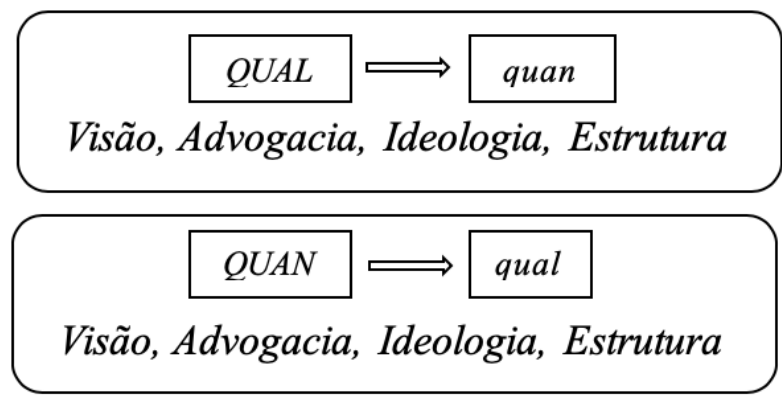

Fonte: Adaptado de Creswell et al. (2003).

A prioridade dos dados pode ser igual ou desigual, sendo que, para sua análise, a integração ocorre na fase de interpretação e discussão (HANSON et al., 2005). Esse design é útil para oferecer aos participantes da pesquisa um melhor entendimento de que determinado fenômeno pode ser mudado a partir do resultado do estudo, além de dar voz a perspectivas ou alternativas diversas (HANSON et al., 2005).

Métodos mistos paralelos ou concorrente: A implementação da coleta de dados abrange opções que consistem em reunir informações ao mesmo tempo (CRESWELL et al., 2007). Assim, ao reunir, de maneira simultânea, as duas formas de dados, procura-se realizar um comparativo das duas formas a fim de buscar achados congruentes, por exemplo, comparar os temas identificados nos dados qualitativos com os resultados estatísticos na análise quantitativa (CRESWELL et al., 2007). Semelhante à pesquisa de métodos mistos de projetos sequenciais, existem três tipos de designs concorrentes: triangulação concorrente, aninhada concorrente e transformativa concorrente (HANSON et al., 2005).

Em projetos de triangulação concorrente, os dados quantitativos e qualitativos são coletados e analisados ao mesmo tempo, 
possuindo igual prioridade, ou seja, a prioridade geralmente é igual para ambas as formas de dados (HANSON et al., 2005). Embora a prioridade seja igual entre os dois métodos, Creswell et al. (2007) afirmam que essa condição se define apenas na aplicação prática, pois a prioridade pode ser dada na abordagem quantitativa ou na qualitativa. A análise de dados é realizada de maneira separada, com a integração acontecendo na fase de interpretação dos dados e, na fase da interpretação, é feita uma discussão sobre até que ponto os dados triangulam ou convergem (HANSON et al., 2005).

Assim, esse design é útil na tentativa de confirmação e validação cruzada, além de corroborar os achados do estudo (GREENE et al., 1989; STECKLER, 1992; HANSON et al., 2005). De acordo com Creswell et al. (2007), esse tipo de projeto é o mais conhecido dos seis principais projetos de métodos mistos.

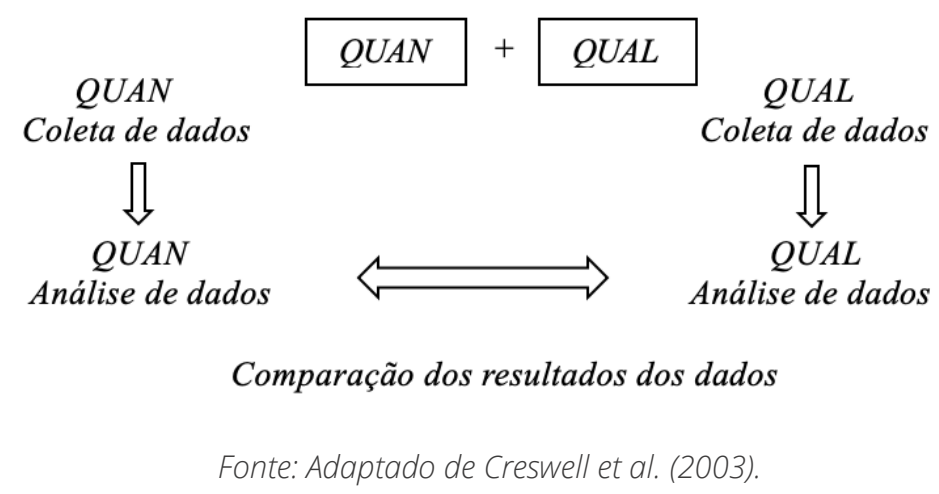

Nos designs de projetos simultâneos aninhados, assim como nos projetos de triangulação concorrentes, os dados quantitativos e qualitativos são coletados e analisados ao mesmo tempo, contudo a prioridade é dada de maneira desigual, sendo voltada para os dados qualitativos ou para os dados quantitativos (HANSON et al., 2005). Assim, esse design possui um método predominante que orientará o projeto, sendo que o método sob menos prioridade (quantitativo ou qualitativo) é aninhado, ou incorporado, dentro do método que for definido como predominante (CRESWELL et al., 2007). 
Dessa forma, esse aninhamento pode significar que a abordagem do método possui uma questão diferente daquela que é adotada pelo método dominante, ou, ainda, pode significar que o método incorporado busque diferentes níveis de informações, assim os dados coletados pelos dois métodos são misturados durante a análise do projeto (CRESWELL et al., 2007).

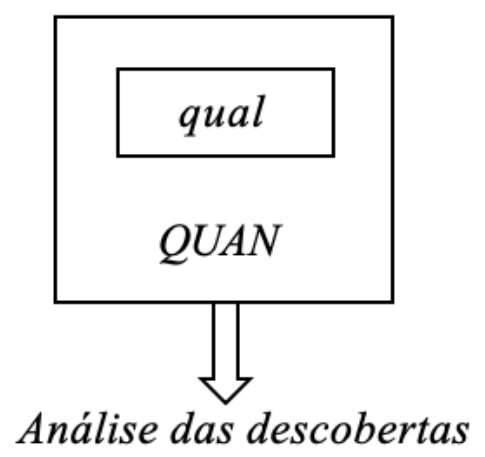

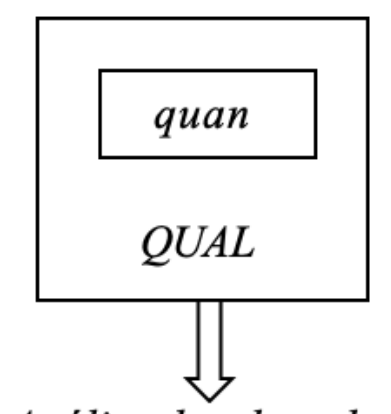

Análise das descobertas

Fonte: Adaptado de Creswell et al. (2003).

Já os projetos concorrentes transformadores utilizam uma lente de defesa explícita, que, geralmente, se reflete nas questões de pesquisa, nas implicações entre ação e mudança e na declaração quanto ao seu propósito (HANSON et al., 2005). Os dados qualitativos e quantitativos são coletados e analisados no mesmo período, sendo que a prioridade pode ser dada de maneira igual ou desigual (HANSON et al., 2005). Ainda, a análise dos dados é realizada de maneira separada, em que a integração desses dados ocorre durante a interpretação deles (HANSON et al., 2005).

Assim como os projetos sequenciais transformadores os projetos concorrentes transformadores são úteis para defender os participantes da pesquisa, oferecendo diversas perspectivas alternativas, além de possibilitar uma melhor compreensão de que determinado fenômeno a ser estudado pode mudar (HANSON et al., 2005). Com isso, o pesquisador utiliza uma perspectiva teórica específica no projeto, sendo que esta perspectiva pode ser defini- 
da com base nas ideologias, além da teoria crítica, da advocacia, da pesquisa participativa (CRESWELL et al., 2007).
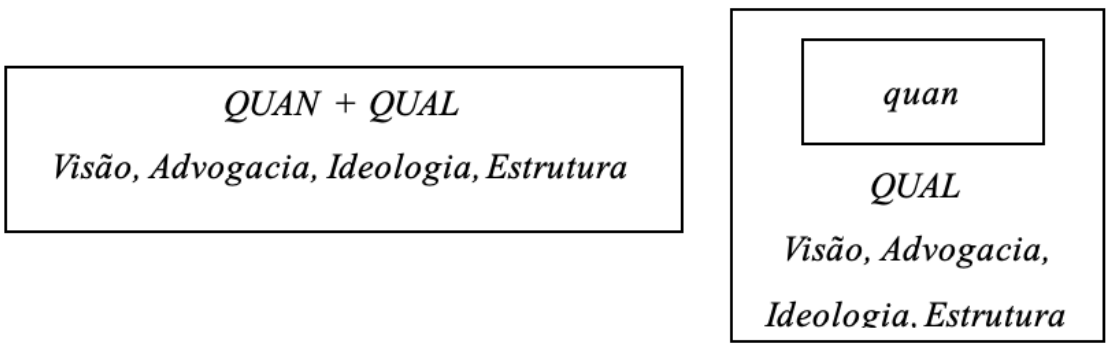

Fonte: Adaptado de Creswell et al. (2003).

Para cada design é definido pelo pesquisador o número de fases, o enfoque com maior peso (quantitativo ou qualitativo), ou, ainda, caso eles recebam a mesma prioridade, é possível definir suas funções e se poderá contar ou não com uma perspectiva teórica e qual seria ela (BROCKE e ROSEMANN, 2013). Assim, para definir o design de um projeto de método misto, é necessário que o pesquisador decida se irá conduzir as fases de maneira concorrente (isto é, independentemente) ou de maneira sequencial (isto é, de forma dependente). Por fim, deve-se analisar o objetivo da mistura dessas abordagens quantitativa e qualitativa (CRESWELL, 2003).

\section{Os Passos básicos para o desenvolvimento de um estudo de métodos mistos em educação física}

Para desenvolver um estudo de métodos mistos é preciso envolver vários passos, muito dos quais se assemelham àqueles seguidos nos métodos tradicionais de pesquisa, que incluem a decisão do objetivo de estudo, as questões da pesquisa e os tipos de dados a serem coletados (HANSON et al., 2005). No estudo de métodos mistos, as partes do estudo são combinadas para a formação de uma totalidade, em que o pesquisador, ou o grupo de 
pesquisa, tem de levar em consideração vários aspectos, que vão desde o planejamento do estudo até a redação do artigo (THOMAS, NELSON e SILVERMAN, 2012). Thomas, Nelson e Silverman (2012) apresentam pontos específicos que devem ser considerados diante da elaboração de um estudo de método misto na área da educação física:

- Perguntas e hipóteses;

- Seleção do delineamento;

- Amostragem;

- Coleta de dados;

- Obtenção de bons dados;

- Apresentação do estudo.

De acordo com Thomas, Nelson e Silverman (2012), algumas dessas decisões são alcançadas de maneira intuitiva, enquanto outras exigem previsões e cooperação entre os pesquisadores envolvidos no estudo. Embora esses passos sejam semelhantes aos métodos tradicionais, Hanson et al. (2005) afirmam que o estudo de método misto envolve ainda ao menos três etapas adicionais. Essas etapas adicionais incluem: decisão do uso de uma lente teórica explícita, identificar quais os procedimentos de coleta de dados e a identificação da análise dos dados e integração, sendo que esses passos ocorrem de forma mais ou menos sequencial (TASHAKKORI e TEDDLIE, 1998; CRESWELL, 1999; HANSON et al., 2005).

Uso de lente teórica explícita: Nessa primeira etapa é decidido se o estudo irá utilizar uma lente teórica, ou seja, se irá utilizar uma base filosófica ou paradigma teórico, por exemplo: pós-positivismo, construtivismo, entre outros; em que subjaz ao estudo e às escolhas metodológicas subsequentes (CROTTY, 1998; HANSON et al., 2005). Como resultado dessa decisão, é informada e influen- 
ciada a metodologia e os métodos que serão utilizados no estudo (HANSON et al., 2005).

Procedimentos para coleta de dados: A segunda etapa trata-se da decisão de como será implementada e priorizada a coleta de dados (HANSON et al., 2005). A implementação é a decisão sobre a forma que os dados quantitativos e qualitativos serão coletados, ou seja, se será de maneira simultânea ou sequencial. Já a prioridade é tida como o peso ou a ênfase relativa que é dada aos dois tipos de dados, sejam eles iguais ou desiguais (CRESWELL, 2003).

Qual ponto ocorrerá a análise de dados e a integração: $A$ terceira etapa trata-se da decisão pelo ponto em que ocorrerão a análise e integração dos dados (HANSON et al., 2005). Nesse estágio, esses processos podem ocorrer através de uma análise dos dados separadamente (dados qualitativos e dados quantitativos). Além disso, transformando ou conectando essas análises de alguma maneira, esse procedimento permite uma visão global do processo multifatorial (TASHAKKORI e TEDDLIE, 1998; ONWUEGBUZIE e TEDDLIE, 2003).

Para a realização de uma pesquisa de método misto os investigadores precisam ser habilidosos nas abordagens qualitativa e quantitativa. Dessa maneira, a participação de peritos em diferentes metodologias pode contribuir com o desenvolvimento da pesquisa. Além disso, esses peritos, na sua área de domínio, podem permitir uma integração de dados mais completa (THOMAS, NELSON e SILVERMAN, 2012).

\section{Conclusão}

O presente artigo apresentou uma introdução aos métodos mistos, além de ter o objetivo de auxiliar na difusão dessa abordagem na área da Educação Física. Assim, a busca pelo entendimento de como se dá o desenvolvimento de um estudo de métodos mistos pode auxiliar o pesquisador diante das construções de pesqui- 
sas e, consequentemente, contribuir para os avanços científicos. O percurso que conduz a realização desse tipo de estudo é marcado pela definição do design da pesquisa, que estabelece os critérios que serão considerados em cada uma das abordagens - qualitativa e quantitativa. Como objetivo final, este estudo apresentou os caminhos e maneiras de se definir o design do estudo de métodos mistos. Para tanto, foram apresentados os esquemas sobre cada design disponível na literatura, de maneira que pudesse facilitar o entendimento do leitor. Este estudo limita-se à possibilidade de melhor detalhar os designs na área da Educação Física, uma vez que esse método carece de atenção específica de outros estudos sobre o tema. Além disso, ainda há poucos trabalhos que integrem as diferentes técnicas de pesquisa na área da Educação Física, embora todos sejam importantes. Nessa conjuntura, recomenda-se a realização de estudos que abordem minunciosamente o percurso metodológico da pesquisa mista na área da educação física.

\section{Referências}

BROCKE, J. V.; ROSEMANN, M. Metodologia de pesquisa. AMGH Editora, 2013.

CAREGNATO, A. F.; ORDONHES, M. T.; CAVICHIOLLI, F. R. Identificação das instalações esportivas e distribuição espacial das principais entidades competitivas do atletismo brasileiro. Revista de Gestão e Negócios do Esporte (RGNE). São Paulo, v. 3, n. 1, 16-34, 2018.

CHERRYHOLMES, C. H. Notes on pragmatism and scientific realism. Educational researcher, EUA, v. 21, n. 6, p. 13-17, 1992.

CRESWELL, J. W. Qualitative inquiry and research design. Thousand Oaks, CA, Sage, 2003.

CRESWELL, J., W. Educational research: Planning, conducting, and evaluating quantitative and qualitative approaches to resear- 
ch. 2nd ed. Upper Saddle River, NJ, Merrill/Pearson Education. 2005.

CRESWELL, J. W.; CLARK, V. L. P. Mixed Methods Search:

Research Methods Series. Publishing Think, 2015.

CRESWELL, J. W.; FETTERS, M. D.; IVANKOVA, N. V. Designing a mixed methods study in primary care. The Annals of Family Medicine, EUA, v. 2, n. 1, p. 7-12, 2004.

CRESSWELL, J. et al. An expanded Typology for classifying mixed methods research into designs. The mixed methods reader. California: sage publications, EUA, p. 159-96, 2007.

CRESWELL, J. W. Mixed-method research: Introduction and application. In: Handbook of educational policy. Academic Press, 1999.

CROTTY, M. The foundations of social research: Meaning and perspective in the research process. Sage, 1998.

DEWEY, J. The later works, 1925-1953. Carbondale, IL, Southern Illinois University Press, v. 6, 1931.

FILGUEIRAS, A. et al. O uso da imagética mental como técnica para melhora do aproveitamento no futebol de campo: um estudo de caso. Revista Coleção Pesquisa em Educação Física, Rio de Janeiro, v. 11, n. 4, p. 167-174, 2012.

GREENE, J. C.; CARACELLI, V. J.; GRAHAM, W. F. Toward a conceptual framework for mixed-method evaluation designs. Educational evaluation and policy analysis, EUA, v. 11, n. 3, p. 255-274, 1989.

HANSON, W. E. et al. Mixed methods research designs in counseling psychology. Journal of counseling psychology, EUA, v. 52, n. 2, p. 224, 2005.

JAARSMA, E. A. et al. Barriers and facilitators of sports in children with physical disabilities: a mixed-method study. Disability and rehabilitation, EUA, v. 37, n. 18, p. 1617-1625, 2014. 
JOHNSON, R. B.; ONWUEGBUZIE, A. J. Mixed methods research: $A$ research paradigm whose time has come. Educational researcher, EUA, v. 33, n. 7, p. 14-26, 2004.

LEECH, N. L.; ONWUEGBUZIE, A. J. A typology of mixed methods research designs. Quality \& quantity, EUA, v. 43, n. 2, p. 265-275, 2009.

MORGAN, D. L. Paradigms lost and pragmatism regained: Methodological implications of combining qualitative and quantitative methods. Journal of mixed methods research, EUA, v. 1, n. 1, p. 48-76, 2007.

MORSE, J. M. Principles of mixed methods and multimethod research design. Handbook of mixed methods in social and behavioral research, EUA, v. 1, p. 189-208, 2003.

ONWUEGBUZIE, A. J.; TEDDLIE, Charles. A framework for analyzing data in mixed methods research. Handbook of mixed methods in social and behavioral research. EUA, v. 2, p. 397-430, 2003. RIDENOUR, C. S; NEWMAN, I. Mixed methods research: Exploring the interactive continuum. SIU Press, 2008.

RALLIS, S. F.; ROSSMAN, G. B. Mixed methods in evaluation contexts: A pragmatic framework. Handbook of mixed methods in social and behavioral research, EUA, p. 491-512, 2003.

SMALL, M. L. How to conduct a mixed methods study: Recent trends in a rapidly growing literature. Annual review of sociology, EUA, v. 37, p. 57-86, 2011.

STECKLER, A. et al. Toward integrating qualitative and quantitative methods: An introduction. Health Education Quarterly, EUA, p. 1-8, 1992.

TASHAKKORI, A.; TEDDLIE, C. Handbook on mixed methods in the behavioral and social sciences. Thousand Oaks, CA, Sage. 2003.

TASHAKKORI, A.; TEDDLIE, C. B. Mixed methodology: Combining qualitative and quantitative approaches. Sage, 1998. 


\title{
Publisher
}

\begin{abstract}
Universidade Federal de Goiás. Faculdade de Educação Física e Dança. Publicação no Portal de Periódicos UFG. As ideias expressadas neste artigo são de responsabilidade de seus autores, não representando, necessariamente, a opinião dos editores ou da universidade.
\end{abstract}

